\title{
Sulfonylurea Use and Cardiovascular Safety Revisited
}

\author{
Javier Morales \\ Associate Clinical Professor of Medicine, Donald and Barbara Zucker School of Medicine at Hofstra/Northwell University, New York
}

${ }^{\star}$ Corresponding author: Javier Morales, Associate Clinical Professor of Medicine, Donald and Barbara Zucker School of Medicine at Hofstra/Northwell University, Vice President, Advanced Internal Medicine Group, East Hills, New York E-mail: Saxodoc@Gmail.com

Received: October 23, 2019; Accepted: November 10, 2019; Published: November 15, 2019;

\begin{abstract}
Sulfonylurea use has been commonplace for the management of type 2 diabetes as an adjunct to metformin over the past decades. Their effectiveness has been repeatedly demonstrated in terms of glycemic control in the short-term however, long-term sustainable control remains in question. Over the years, FDA mandated cardiovascular safety trials have been completed involving most newer antidiabetic therapies to the market place however, the sulfonylurea class had not been studied until the recent head-to-head cardiovascular outcomes trial involving the comparison of linagliptin, an inhibitor of DPP-IV, with glimepiride in the CARMELINA study where non-inferiority was demonstrated in both treatment groups. While this finding seems to be reassuring, does it really confer safety of use of sulfonylurea drugs in the management of type 2 diabetes?
\end{abstract}

Keywords: Sulfonylurea, DPP-IV inhibitor, diabetes, cardiovascular disease, major adverse cardiovascular event, type 2 diabetes, hypoglycemia, arrhythmia

\section{Guidelines}

Since the evolution of the management of diabetes and hyperglycemia, respected societies globally have been providing guidance with respect to such management. Since the availability of such drugs, the class of sulfonylurea was adapted and implemented. After metformin was approved for use, the sulfonylurea was recognized as the second agent for intensification. Over recent years and on the basis of newer agents which demonstrated safety that have become available, namely incretin agents and urinary SGL T2 inhibitors, the sulfonylurea class has found its way on the bottom of such algorithms, as per the American Association of Clinical Endocrinologists [1] on the basis of safety and efficacy, and as one of the executable options of those financially challenged, according to the joint consensus statement from the American Diabetes Association and European Association for the Study of Diabetes, recently revised in the first quarter of 2019 [2]. While these statements and guidelines are consensus or expert based, they are recommendations that are soundly based on their demonstrated safety and efficacy, but even then, the choice of not following such recommendations is routinely exercised.

\section{Development of sulfonylurea}

The first agents that were discovered in the sulfonylurea class was in 1942 where sulfonamides were noted to reduce blood sugar in non-human studies, leading way to the development of Carbutamide, which was very quickly withdrawn from the market place because of apparent hematologic disease, particularly on the bone marrow [3]. Second-generation sulfonylureas that became available differed from their first-generation counterparts because of differences in absorption and metabolism. For this reason, the second-generation agents have been credited with fewer hypoglycemic events relative to their first-generation counterparts, but still differ greatly based on molecular formulation whereas glimepiride is noted to produce hypoglycemia in $2 \%$ to $4 \%$ patients compared to glyburide, noted to produce hypoglycemia in $20-30 \%$ of patients with the reason being better preservation of prevention of insulin secretion and promotion of glucagon secretion [6].

\section{Mechanism of action}

The mechanism of action described as that of insulin secretion out of the pancreatic beta cell independent of what the blood glucose level in circulation may be in addition to having a decreased effect on hepatic insulin clearance. This insulin secretory effect is largely as a consequence of blocking potassium inflow into the cells through a DPP dependent channel. This leads to membrane repolarization leading to increase cellular inflow of calcium into these beta cells leading to filamentous contraction of actinomysin with subsequent secretion of large quantities of insulin from that beta cell. While insulin is secreted in 2 phases largely, it appears that the effect of the sulfonylurea tends to be more so on the second phase of secretion. However, review of the literature demonstrates that there might be down-regulation of sulfonylurea receptors on the surface of beta cells with long-term use, with increased expression of those very receptors after discontinuation of treatment with sulfonylurea over a certain period of time [3].

\section{Sustainability}

Large-scale clinical trials have been performed over the years to evaluate the development of microvascular and macrovascular complications associated with the management of patients with type 2 diabetes. Amongst these were the United Kingdom Prospective Diabetes Study [4] and the ADOPT trial [5]. In both of these trials, different agents were studied that included insulin, the sulfonylurea 
group, and metformin. The p-par gamma molecule, rosiglitazone, was also studied in the ADOPT trial. It was interesting to note that in these 2 large-scale trials, the sulfonylurea class led to a rapid reduction in hemoglobin Alc that seemed to worsen by about the second year of therapy, or thereafter with a subsequent rise suggesting treatment failure. Progressive dysfunction and worsening insulin secretion has been noted with sulfonylurea use despite better glycemic control in the short-term. This phenomenon has been labeled as secondary failure and is an outcome shortly to be expected with chronic sulfonylurea use. While not terribly well understood, and as mentioned above, is likely related to down-regulation of sulfonylurea receptors on beta cell surface membrane [3]. Thus there appears to be multiple factors that might be contributing to lack of sustainability in hemoglobin A1c control and these stem from increasing pressures that lead to accelerated apoptosis or cell death, and other mechanisms yet to be discovered that may perhaps be implemented in the future for beta cell preservation. Thus on the basis of demonstrated lack of sustainability of hemoglobin Alc, one can assume that treatment with a sulfonylurea would offer very little on beta cell mass preservation or persistent improvement in beta cell function.

\section{Safety}

Use of any pharmacologic agent for management of chronic disease may have adverse events associated with them, even though they may be curtailing the natural history of the original disease state. For the sulfonylurea class, however, the most worrisome challenges include progressive weight gain, as evidenced in numerous large-scale clinical trials, and the risk of developing significant hypoglycemia, which itself is challenging in diagnosing, particularly nocturnal hypoglycemia, which often goes unrecognized. When reviewing the literature, there has been significant variability in nocturnal hypoglycemia listed ranging anywhere from $20-40 \%$ depending on which study was reviewed and with which sulfonylurea. However, what is the cost of hypoglycemia? From a physiologic standpoint, significant electrolyte aberrancies can occur including potassium shifting intracellularly as well as effect on the myocyte cycle with noted QT prolongation [7]. Such occurrences can lead to significant dysrhythmia and lethal arrhythmia. It is thought that such unpredictable variability in glycemic control may have been part of the reason why an increased mortality may have been observed in the ACCORD Action to Control Cardiovascular Risk in Diabetes) trial where the forced titration hemoglobin Alc target was a value of less than or equal to $6 \%$. It is interesting to note that the majority of the cardiovascular events recorded were in the population of patients who is hemoglobin Alc did not change very much despite aggressive management, suggesting much glycemic variability [12].

\section{Cardiovascular outcomes}

Several studies have been published looking at particular cardiovascular adverse events with the use of sulfonylureas. The data seems to be quite variable. Data reviewing the UK Clinical Practice Research Data Link, published in 2017, reviewed short acting and nonspecific long-acting sulfonylurea with no significant increase in noted myocardial infarction, ischemic stroke or cardiovascular death between both long and short acting agents however, with significant risk of severe hypoglycemia in the long-acting agents [8]. In another study accepted for publication in June 2018 assessed whether adding or switching to sulfonylurea is associated with an increased risk of major adverse cardiovascular events including all-cause mortality. This study did demonstrate an increased risk of myocardial infarction and all-cause mortality, with no differences in cardiovascular death or severe hypoglycemia [9].

As part of the management of diabetes, which is complex already to begin with, newer agents with lower hypoglycemic potential when used as monotherapy or combination therapy with metformin have gained significant traction on the basis of their safety record demonstrating no increased cardiovascular risk or reduction in cardiovascular risk. Such therapies include DPP 4 inhibitors, GLP1 receptor agonists, and urinary SGL T2 receptor blockers. Only recently has a cardiovascular outcomes trial been completed where the DPP 4 inhibitor linagliptin was studied with the active comparator being the sulfonylurea glimepiride in the CAROLINA trial [10]. The purpose of this trial was to establish noninferiority between these 2 agents with respect to cardiovascular risk. However, since no cardiovascular studies have been performed looking at glimepiride, cardiovascular safety was demonstrated with the DPP 4 Linagliptin versus placebo in the CARMELINA study where noninferiority was achieved [11]. In the active comparator CAROLINA (CARdiovascular Outcome study of LINAgliptin versus glimepiride in patients with type 2 diabetes) study, the primary endpoint defined as noninferiority of linagliptin versus glimepiride in time to first occurrence 3 point MACE was satisfied. The study was an event driven trial involving 6979 patients with the median duration of the study being 2.2 years. Population involved was on average 62 years of age with $34 \%$ having had established cardiovascular disease and $28.6 \%$ of those in the trial having been treated with a sulfonylurea agent for less than 5 years. Noninferiority for major adverse cardiovascular events was indeed demonstrated, albeit with significantly greater hypoglycemia noted in the glimepiride treatment group (10.6\% versus $37.7 \%)$.

\section{Conclusion}

Sulfonylurea use over the past decades has been welcomed by a sense of comfort and demonstrated rapid efficacy, although of limited benefit. Weight gain and hypoglycemia still seems to be the most worrisome adverse events with these agents, and a myriad of physiologic effects as a consequence of those hypoglycemic events will pose significant challenges toward their continued use. While electrolyte shifting and effects on QT intervals increase risk of cardiac arrhythmia, there was no increase in cardiovascular mortality that was noted in the glimepiride subgroup, treated to a maximum of 4 mg daily, in the CARMELINA study. Of note was the fact that those enrolled in the clinical trial was a lower cardiovascular risk population, albeit older. The fact that there was no increase in cardiovascular events noted in this clinical trial was reassuring but should not be translated to the sulfonylurea class in general as only glimepiride use was allowed by trial design. The observed risk reduction cannot be and should not be extrapolated to other sulfonylureas, and while safety was demonstrated from a cardiovascular standpoint in this low risk 
population, there exists uncertainty of whether or not similar findings would be seen in a higher risk population. Therefore, it's important for the prescriber to be aware that differences in this class of agents need to be taken into consideration in order to avoid a false sense of reassurance.

\section{List of abbreviations}

UKPDS- United Kingdom Prospective Diabetes Study

ADOPT- A Diabetes Outcome Progression Trial

GLP-1 receptor agonist-glucagon-like peptide 1 receptor agonist

SGL T2 receptor inhibitor-sodium glucose transport protein 2 inhibitor

SU-sulfonylurea

DPP 4 or DPP IV-Dipeptidyl peptidase 4

MACE Major Adverse Cardiovascular Events

\section{Declaration}

\section{Compliance with Ethics Guidelines}

This article is based on previously conducted studies and does not contain any studies with human participants or animals performed by any of the offers of this publication

\section{Consent for publication}

The author has given his approval for the version of this manuscript to be published

\section{Competing interests/Disclosures}

Dr. Javier Morales is on the speakers Bureau of Novo-Nordisk, Eli Lilly and company, Boehringer Ingelheim, Janssen pharmaceuticals, Mylan pharmaceuticals, and Abbott Laboratories, and serves as consultant, as well as having participated in advisory board meetings for the above-named entities.

\section{Authorship}

The author meets the International Committee of Medical General Editors (ICMJE) criteria for authorship for this article, take responsibility for the integrity of the work as a whole

\section{Availability of data and material}

This article is based on previously conducted studies and thorough literature review was conducted during authorship of this manuscript

\section{Funding}

No funding or sponsorship was received for this publication or article processing charges

\section{Acknowledgments}

No editorial assistance was provided by any entity or company during the development of this manuscript.

\section{References}

1. Alan J Garber, Martin J Abrahamson, Joshua I Barzilay, Lawrence Blonde, Zachary T Bloomgarden, et al. (2019) Consensus Statement By The American Association Of Clinical Endocrinologists And American College Of Endocrinology On The Comprehensive Type 2 Diabetes Management Algorithm - 2019 Executive Summary. Endocrine practice 25: 1

2. Introduction: Standards of Medical Care in Diabetes (2019) Diabetes Care. 42: 1-2.

3. Daniele Sola, Luca Rossi, Gian Piero Carnevale Schianca, Pamela Maffioli, Marcello Bigliocca, et al. (2015) Sulfonylureas and their use in clinical practice. Arch Med Sci 11: 840-848.

4. UK Prospective Diabetes Study (UKPDS) Group (1998) Effect of intensive bloodglucose control with metformin on complications in overweight patients with type 2 diabetes (UKPDS 34). The Lancet 352: 854-865.

5. Giancarlo Viberti, Steven E. Kahn, Douglas A Greene, William H Herman, Bernard Zinman, Rury R Holman, et al. (2002) A Diabetes Outcome Progression Trial (ADOPT). Diabetes Care 25: 1737-1743.

6. Mark T Keegan (2013) Pharmacology and Physiology for Anesthesia

7. Laitinen T, et al. (2008) Ann Noninvasive Electrocardiology 13: 97-105.

8. Antonios Douros, Hui Yin, Oriana Hoi Yun Yu, Kristian B. Filion, Laurent Azoulay and Samy Suissa (2017) Pharmacologic Differences of Sulfonylureas and the Risk of Adverse Cardiovascular and Hypoglycemic Events. Diabetes Care 40: 15061513 .

9. Antonios Douros, Sophie Dell'Aniello, Oriana Hoi Yun Yu, Kristian B Filion, Laurent Azoulay, et al. (2018) Sulfonylureas as second line drugs in type 2 diabetes and the risk of cardiovascular and hypoglycaemic events: population based cohort study 362

10. CAROLINA: Cardiovascular Outcome Study of Linagliptin versus Glimepiride in Patients with Type 2 Diabetes. ClinicalTrials.gov

11. Rosenstock J, Perkovic V, Johansen OE, Cooper ME, Kahn SE, Marx N, et al. (2019) Effect of Linagliptin vs Placebo on Major Cardiovascular Events in Adults With Type 2 Diabetes and High Cardiovascular and Renal Risk: The CARMELINA Randomized Clinical Trial. JAMA 321: 69-79.

12. Effects of Intensive Glucose Lowering in Type 2 Diabetes (2008) N Engl J Med 358: 2545-2559

\section{Citation:}

Javier Morales (2019) Sulfonylurea Use and Cardiovascular Safety Revisited. Endocrinol Diabetes Metab J Volume 3(6): 1-3. 